\title{
Daptomycin in treatment of Methicillin-resistant Staphylococcus aureus diskitis
}

\author{
Ciro Parlato • Giuseppe di Nuzzo • Marianna Luongo • \\ Silvano Esposito $\cdot$ Aldo Moraci
}

Received: 17 December 2009/ Accepted: 1 February 2011/Published online: 16 February 2011

(C) The Author(s) 2011. This article is published with open access at Springerlink.com

Sir,

Daptomycin is a new lipopeptide antibiotic with potent activity against Methicillin-resistant Staphylococcus aureus. It is a bactericidal agent that has the potential to treat infections caused by multi-drug-resistant grampositive organisms. Although the mainstay of treatment is based on glycopeptides, treatment with vancomycin is frequently reported as often unsuccessful [1-3]. We describe a case of Methicillin-resistant Staphylococcus aureus (MRSA) diskitis successfully treated with intravenous daptomycin. A 38-year-old man was admitted to our department for lumbar pain and fever. Other surgeons have submitted the patient to surgery for L5-S1 discectomy and they discharged him with empirical treatment of ceftriaxone ( $2 \mathrm{~g} /$ day). At admission to our department, he had no notable examination findings, except for fever of $39.2^{\circ} \mathrm{C}$. CT revealed typical findings of diskitis (Fig. 1a) and MRSA was isolated from blood cultures. We did not perform surgical operation and preferred conservative treatment. Because of the patient's history of vancomycin allergy, daptomycin treatment was

C. Parlato $(\bowtie) \cdot$ G. di Nuzzo •

M. Luongo - A. Moraci

Department of Neurosurgery,

Second University of Naples, Viale Colli Aminei 21,

80131 Naples, Italy

e-mail: ciro.parlato@unina2.it

S. Esposito

Department of Infectious Diseases,

Second University of Naples, Naples, Italy initiated (6 mg/kg every $24 \mathrm{~h}$ i.v.) and treatment with other antibiotics was discontinued. Erythrocyte sedimentation rates and serum C-reactive protein levels returned to the preoperative levels, within 15 days after the initiation of the treatment. Additional blood cultures showed no growth after antibiotic therapy was initiated, and daptomycin was tolerated by patient without treatmentrelated adverse events. Results of follow-up cultures showed bacterial eradication, nevertheless, we decided to stop the therapy, when MR and scintigraphy with labeled leucocytes were normal to avoid the return of the infection and we needed to continue the daptomycin therapy for 92 days; he returned to his work, without any residual neurologic deficits (Fig. 1b, c). The usual therapeutic choice in cases of MRSA infections is a glycopeptide with or without rifampin, but increasing evidence indicates decreasing effectiveness of vancomycin for Staphylococcus aureus infection. [1-3]. For patients who develop a reaction to vancomycin, treatment of MRSA infections remains clinically challenging. Daptomycin has potent bactericidal activity against gram-positive bacteria, i.e., concentration-dependent. Unlike agents that are active against the cell wall, daptomycin causes rapid bactericidal activity without cell lysis, which could reduce the release of bacterial molecules and lessen the inflammatory response [1-3]. The US Food and Drug Administration has approved daptomycin for the treatment of complicated skin and skin structure infections caused by susceptible pathogens as well as for bacteremia, including right-sided endocarditis, caused by MRSA [1-4]. Other authors have reported cases of severe myopathy and acute renal failure related to daptomycin $[4,5]$. Our case suggests that daptomycin is a safe and effective option for the treatment of MRSA diskitis, without treatment-related adverse events. 
Fig. 1 a CT showing typical findings of discitis after left interlaminar approach for L5-S1 discectomy. b MR showing no inflammatory findings, after daptomycin therapy.

c Scintigraphy with labeled leucocytes showing normal findings, on this data we decided to stop the therapy
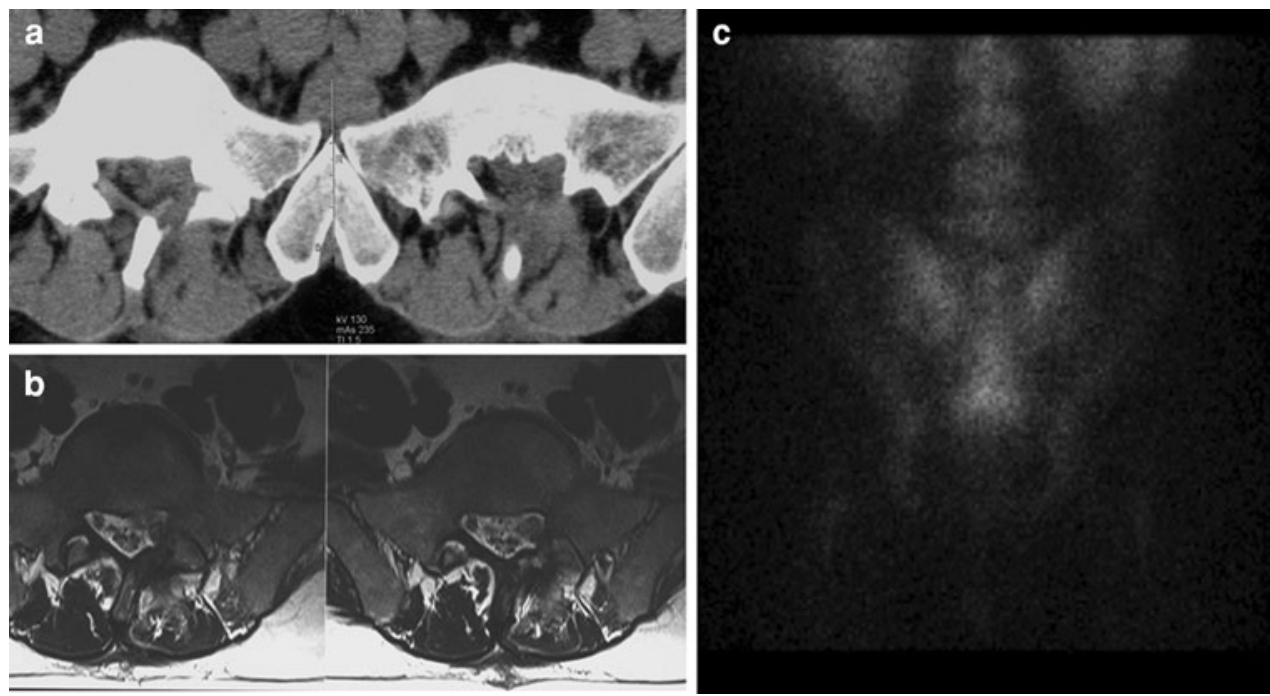

Open Access This article is distributed under the terms of the Creative Commons Attribution Noncommercial License which permits any noncommercial use, distribution, and reproduction in any medium, provided the original author(s) and source are credited.

\section{References}

1. Antony SJ (2006) Combination therapy with daptomycin, vancomycin, and rifampin for recurrent, severe bone and prosthetic joint infections involving methicillin-resistant Staphylococcus aureus. Scand J Infect Dis 38(4):293-295

2. Bassetti M, Mikulska M, Schenone E, Nicolini L, Viscoli C (2009) Long course of daptomycin in the treatment of meticillin-resistant
Staphylococcus epidermidis endocarditis and spondylodiskitis. Int J Antimicrob Agents 34(3):290-291

3. Burdette SD (2009) Daptomycin for methicillin-resistant Staphylococcus aureus infections of the spine. Spine J 9(6):e5-e8

4. Kazory A, Dibadj K, Weiner ID (2006) Rhabdomyolysis and acute renal failure in a patient treated with daptomycin. J Antimicrob Chemother 57:578-579

5. Marty FM, Yeh WW, Wennersten CB, Venkataraman L, Albano E, Alyea EP, Gold HS, Baden LR, Pillai SK (2006) Emergence of a clinical daptomycin-resistant Staphylococcus aureus isolate during treatment of methicillin-resistant Staphylococcus aureus bacteremia and osteomyelitis. J Clin Microbiol 44(2):595-597 